\title{
Nucleon Partonic Spin Structure to be Explored by the Unpolarized Drell-Yan Program of COMPASS Experiment at CERN
}

\author{
Wen-Chen Chang \\ on behalf of COMPASS Collaboration \\ Institute of Physics, Academia Sinica, Taipei 11529, Taiwan \\ changwc@phys.sinica.edu.tw \\ Published 29 February 2016
}

\begin{abstract}
The observation of the violation of Lam-Tung relation in the $\pi N$ Drell-Yan process triggered many theoretical speculations. The TMD Boer-Mulders functions characterizing the correlation of transverse momentum and transverse spin for partons in unpolarized hadrons could nicely account for the violation. The COMPASS experiment at CERN will measure the angular distributions of dimuons from the unpolarized Drell-Yan process over a wide kinematic region and study the beam particle dependence. Significant statistics is expected from a successful run in 2015 which will bring further understanding of the origin of the violation of Lam-Tung relation and of the partonic transverse spin structure of the nucleon.
\end{abstract}

Keywords: Drell-Yan process, Lam-Tung relation, Boer-Mulders function, partonic spin structure.

\section{Introduction}

The continuum of dilepton from hadron-hadron collisions has been successfully described by the Drell-Yan parton model $^{1}$ where collinear quarks and antiquarks $(q \bar{q})$ from two individual hadrons electromagnetically annihilate into a virtual photon. The predictions of the scaling behavior, the $A$-dependence of production cross section, and the polar angle distribution of leptons have been experimentally verified. ${ }^{2,3}$ Furthermore the quantitative description of the transverse momentum distribution of lepton pairs and the enhancement of the cross section over the naive Drell-Yan estimate do call for the perturbative QCD effect of gluon emission and absorption by the partons.

Drell-Yan process has become one of the most intensively studied processes in $\mathrm{QCD}^{4,5}$ and an effective tool to learn about the partonic structure of hadrons, ranging from the parton distribution functions (PDFs) to transverse momentum

This is an Open Access article published by World Scientific Publishing Company. It is distributed under the terms of the Creative Commons Attribution 3.0 (CC-BY) License. Further distribution of this work is permitted, provided the original work is properly cited. 
dependent (TMD) parton density distributions. Nonetheless there remain challenges to understand why Lam-Tung relation failed in the dilepton azimuthal angular distributions of the $\pi N$ Drell-Yan reaction. ${ }^{6}$

Since 2002 the COMPASS collaboration at CERN has been one of the major experiments for studying both unpolarized and polarized parton structures of nucleons, and TMD distributions. ${ }^{7}$ Nonzero TMD Sivers functions for the valence quarks of nucleons have been measured in semi-inclusive DIS (SIDIS). ${ }^{8}$ In the second phase of the experiment, ${ }^{9}$ COMPASS will seek for the Sivers functions in the first-ever polarized Drell-Yan experiment with a transversely-polarized $\mathrm{NH}_{3}$ target. ${ }^{10}$ This measurement will be an important experimental test of the long-predicted sign change of Sivers functions in polarized SIDIS and Drell-Yan processes.

In this talk we report the impact on TMD spin physics to be brought by the simultaneous measurement of the unpolarized Drell-Yan process in COMPASS. The violation of Lam-Tung relation in the $\pi N$ Drell-Yan process and the interpretation of this phenomenon by TMD Boer-Mulders functions and other theoretical models will be introduced. A comprehensive measurement in the coming unpolarized DrellYan program of CERN/COMPASS experiment shall shed light on this problem.

\section{Angular Distributions of Unpolarized Drell-Yan Process}

Assuming dominance of the single-photon process, the angular distribution of leptons from the unpolarized Drell-Yan process could be expressed by $d \sigma / d \Omega \propto$ $\left(1+\lambda \cos ^{2} \theta+\mu \sin 2 \theta \cos \phi+\frac{\nu}{2} \sin ^{2} \theta \cos 2 \phi\right)$, where $\theta$ and $\phi$ are the polar and azimuthal angles of the decay leptons in the virtual photon rest frame, and $\lambda, \mu$ and $\nu$ are angular parameters. The virtual photon from collinear $q \bar{q}$ annihilation is transversely polarized and thus the decay angular distribution of muons is proportional to $\left(1+\cos ^{2} \theta\right)$, i.e. $\lambda=1$ and $\mu=\nu=0$. The early experimental measurement agreed with this prediction.

In 1978, Lam and Tung studied the quark intrinsic transverse momentum effects on the angular distributions of lepton pairs. ${ }^{11}$ They found that QCD effect could lead to $\lambda \neq 1$ and $\mu, \nu \neq 0$ but the relation $1-\lambda=2 \mu$, the so-called "LamTung relation" holds for NLO QCD effect. Therefore this relation provides a unique opportunity to test the "QCD-improved quark-parton model". ${ }^{11}$

The very first measurement by the $\mathrm{NA}^{12}$ experiment showed that $\nu$ increases strongly toward large transverse momentum of the lepton pair $\left(p_{T}\right)$ but the LamTung relation was roughly preserved. Nevertheless the following measurements with better statistics by the NA10 ${ }^{13}$ and the E615 ${ }^{14}$ experiments clearly identified a strong violation of Lam-Tung relation in the $\pi N$ Drell-Yan process with nuclei and deuterium targets. The degree of violation got stronger for dimuon pairs with large $p_{T}$.

The nonzero $\nu$ or $\cos 2 \phi$ asymmetry comes from the interference of virtual photon amplitudes with opposite helicities. ${ }^{15,16}$ The occurrence of " Lam-Tung violation" suggests certain mechanisms leading to the correlation of the helicities of quark and 
Table 1. Theoretical interpretations of Lam-Tung violation in the $\pi N$ Drell-Yan process.

\begin{tabular}{cccc}
\hline & $\begin{array}{c}\text { Boer-Mulders } \\
\text { Function }\end{array}$ & QCD & Glauber gluon \\
& chromo-magnetic effect & \\
origin of effect & hadron & QCD vacuum & pion specific \\
quark flavor dependence & yes & no & no \\
beam dependence & yes & no & yes \\
large $p_{T}$ limit & 0 & finite & 0 \\
\hline
\end{tabular}

anti-quark from the two individual hadrons. Such an effect is commonly expected to be of nonperturbative QCD nature.

Brandenburg et al. ${ }^{15}$ proposed a factorization breaking effect caused by nontrivial QCD vacuum where the chromo-magnetic Sokolov-Ternov effect introduced a spin correlation between the annihilating quark and anti-quark. ${ }^{17}$ On the other hand, Boer ${ }^{18}$ considered a hadronic effect of the spin-orbit correlation of transversely polarized noncollinear partons inside an unpolarized hadron, "Boer-Mulders functions" $\left(h_{1}^{\perp}\right),{ }^{19}$ and the angular parameter $\nu \propto h_{1}^{\perp}\left(q_{N}\right) h_{1}^{\perp}\left(\bar{q}_{\pi}\right)$. Recently it was shown that the $p_{T}$ dependence of $\nu$ measured by NA10 and E615 experiments could be reasonably described by the Boer-Mulders functions and the parton density of pions and nucleons constructed within the light-front constituent model. ${ }^{20}$

Table 1 lists the predictions given by the various theoretical models including one considering Glauber gluons in the $k_{T}$ factorization theorem. ${ }^{21}$ It is clear that a comprehensive measurement of dilepton angular distributions with different types of beam like $\pi^{ \pm}, p, K^{ \pm}$and $\bar{p}$ over a wide kinematic range is required to clarify the mechanisms. For example, the lack of Lam-Tung violation in the $p N$ Drell-Yan process $^{22}$ could be explained by a small Boer-Mulders function for sea quarks in the target nucleon but might be less compatible with the supposedly flavor-blind QCD vacuum effect.

\section{Unpolarized Drell-Yan Program of COMPASS Experiment at CERN}

As illustrated in Fig. 1, the COMPASS experiment is located at the SPS M2 secondary beam line at CERN. The secondary beam produced by the slow-extracted $400 \mathrm{GeV} / \mathrm{c}$ primary proton beam can be either muon or hadron secondary beams in a momentum range of $50 \mathrm{GeV} / \mathrm{c}$ to $280 \mathrm{GeV} / \mathrm{c}$. For $190 \mathrm{-GeV}$ momentum, the negatively-charged hadron beam is composed of $97 \% \pi^{-}, 2.5 \% K^{-}$and $1 \% \bar{p}$ while it is $75 \% p$ and $24 \% \pi^{+}$for the positively-charged beam. The flux of the $\pi^{-}$beam is $10^{8} / \mathrm{s}$. There is a Cerenkov differential counter (CEDAR) in front of the target which can provide particle identification of incident beam. The CEDAR detector will enable the measurement of kaon and antiproton-induced Drell-Yan processes in COMPASS.

In order to detect the dimuon pairs from high-energy Drell-Yan processes, a hadron absorber is required to prevent copious amounts of produced hadrons from 


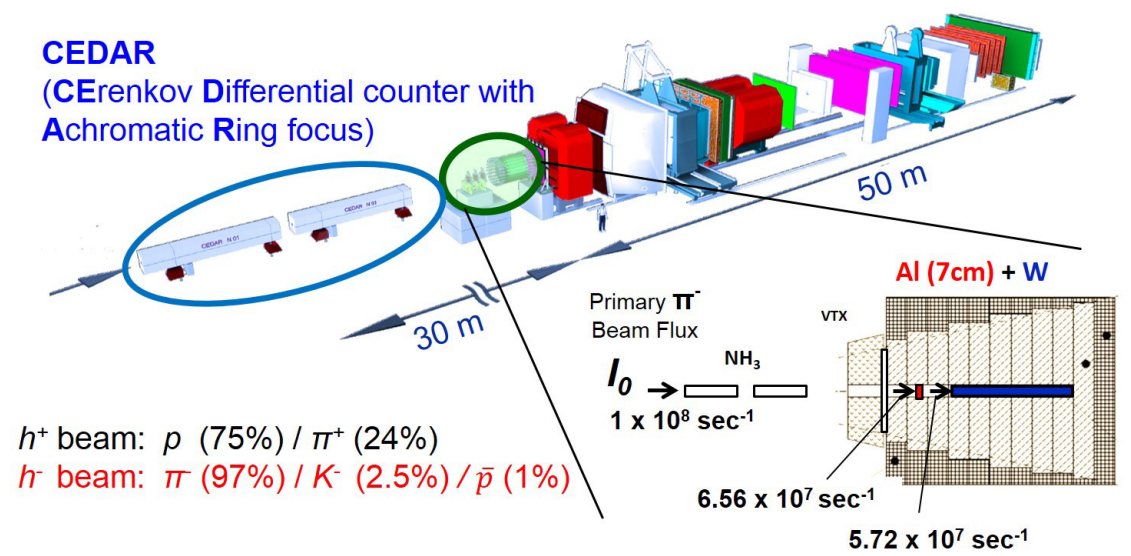

Fig. 1. The beam composition, detector setup and hadron absorber of COMPASS Drell-Yan experiment.

entering the spectrometer. The COMPASS hadron absorber was made of aluminum and alumina $\left(\mathrm{Al}_{2} \mathrm{O}_{3}\right)$ with a downstream $20 \mathrm{~cm}$ layer of steel. It was designed to optimize both the stopping power for hadron and the multiple scattering effect on muons. Inside the absorber, a long tungsten (W) beam plug and a $7 \mathrm{~cm}$ aluminum (Al) plate are placed. Both serve as nuclear targets for the unpolarized Drell-Yan process.

The detector system is composed of a two-stage spectrometer such that lowmomentum charged particles in sideward direction as well as high-momentum particles in forward direction could be well accepted. Therefore COMPASS supersedes the past E615 experiment by one order of magnitude in the overall acceptance of $40 \%$, especially that at large $p_{T}$ region where the Boer-Mulders functions are more visible.

The feasibility of Drell-Yan measurement in COMPASS has been checked by several test runs. In 2009 there was a 3-day test run where two 40-cm polyethylene cylinders and a prototype hadron absorber were implemented. About $6000 \mathrm{~J} / \psi$ were reconstructed from the data as seen in Fig. 2. The measured yield agreed nicely with the one expected from Monte-Carlo simulation. The high-mass Drell-Yan continuum was clearly observed.

Assuming a beam flux of $10^{8} / \mathrm{s}$ for the $\pi^{-}$beam, the expected statistics of unpolarized high-mass $\left(4<M_{\mu \mu}<9 \mathrm{GeV}\right)$ Drell-Yan events from the three targets $\left(\mathrm{NH}_{3}, \mathrm{Al}\right.$ and $\left.\mathrm{W}\right)$ in a 140 -day beam time are $800 \mathrm{k}, 10 \mathrm{k}$ and $6 \mathrm{k}$ for $\pi, K$ and $\bar{p}$-induced Drell-Yan events respectively. Compared to the statistics from NA3, ${ }^{12}$ NA10, ${ }^{13}$ E537 ${ }^{23}$ and E615, ${ }^{14}$ a successful Drell-Yan experiment in 2015 will increase the existing statistics of $\pi, K$ and $\bar{p}$-induced Drell-Yan by a factor of 10 . Fig. 3 shows the expected sensitivity of the angular parameter $\nu$ as a function of $p_{T}$ for $\pi, K$ and $\bar{p}$-induced Drell-Yan events from $\mathrm{W}$ target in COMPASS. Compared to NA10, the statistical error for Drell-Yan data with $\pi^{-}$beam will be significantly 


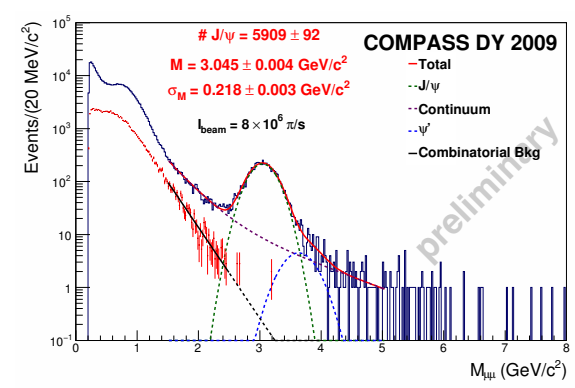

(a)

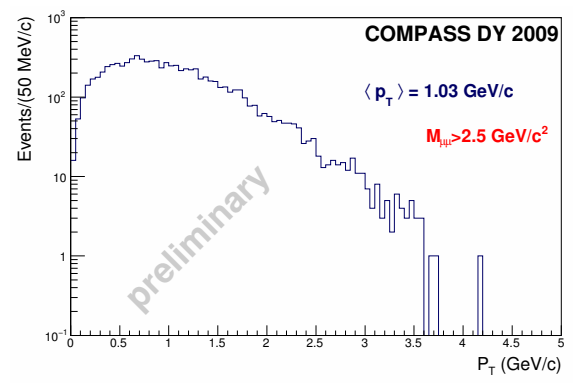

(b)

Fig. 2. (a) Invariant mass and (b) transverse momentum distributions of dimuon events from COMPASS 2009 test run.

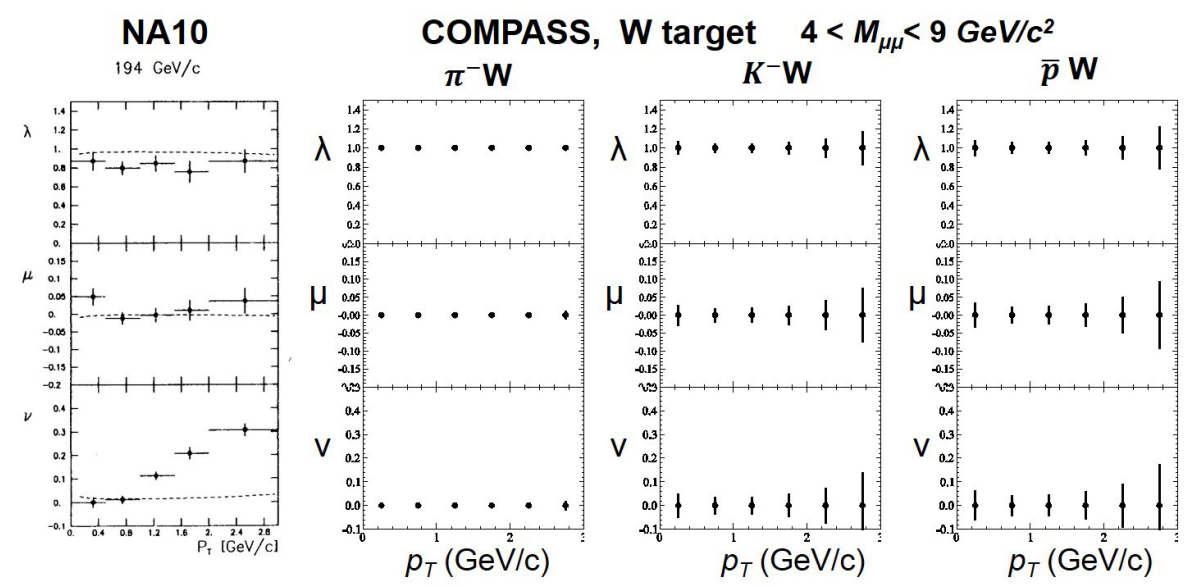

Fig. 3. Expected sensitivity of the angular parameters $\lambda, \mu$ and $\nu$ as a function of $p_{T}$ for $\pi^{-}$, $K^{-}$and $\bar{p}$ beam with $\mathrm{W}$ target in COMPASS, compared with NA10's results for $\pi^{-}$beam. ${ }^{13}$

reduced and a reasonable sensitivity could be achieved for data with $K^{-}$and $\bar{p}$ beam.

Commissioning of COMPASS Drell-Yan experiment was already completed during mid-October - December, 2014. The physics run will take place in year 2015. The beam time in 2016 and 2017 will be reserved for DVCS program. Supposing that the LHC RUN2 is extended, there will be possibility of one extra year for polarized (and unpolarized) Drell-Yan run in 2018. For the long-term plan for Drell-Yan program in COMPASS, polarized ${ }^{6} \mathrm{LiD}$ and long $\mathrm{LH}_{2}$ targets and high intensity RF-separated antiproton/kaon beam is considered. 


\section{Summary}

The TMD Boer-Mulders functions characterizing the spin-orbit correlation of transversely polarized partons offer a reasonable interpretation of the violation of LamTung relation observed in the $\pi N$ DY process. The COMPASS experiment will study not only the Sivers functions in the polarized Drell-Yan process but also the Boer-Mulders functions in the unpolarized DY process using $\pi, K$ and $\bar{p}$ beams over a wide kinematic range. A successful run in 2015 will hopefully bring further understanding of the origin of Lam-Tung violation and the partonic structures of protons and pions.

\section{References}

1. S.D. Drell and T.M. Yan Phys. Rev. Lett. 25, 316 (1970); S.D. Drell and T.M. Yan Phys. Rev. Lett. 25, 902 (1970).

2. I.R. Kenyon, Rep. Prog. Phys. 45, 1261 (1982).

3. C. Grosso-Pilcher and M.J. Shochet, Annu. Rev. Nucl. Part. Sci. 36, 1 (1986).

4. P.L. McGaughey, J.M. Moss, and J.C. Peng, Annu. Rev. Nucl. Part. Sci. 49, 217 (1999).

5. J.C. Peng and J.W. Qiu, Prog. Part. Nucl. Phys. 76, 43 (2014).

6. W.C. Chang and D. Dutta, Internat. J. Modern Phys. E 22, 1330020 (2013).

7. Talk at SPIN2014 by M. Stolarski, http://goo.gl/xzTdxb.

8. Talk at SPIN2014 by B. Parsamyan, http://goo.gl/TKfecf.

9. COMPASS-II Proposal, CERN-SPSC-2010, http://cds.cern.ch/record/1265628.

10. Talk at SPIN2014 by B. Parsamyan, http://goo.gl/zWQ7Go.

11. C.S. Lam and W.K. Tung, Phys. Rev. D 18, 2447 (1978); C.S. Lam and W.K. Tung, Phys. Lett. B 80, 228 (1979); C.S. Lam and W.K. Tung, Phys. Rev. D 21, 2712 (1980).

12. J. Badier et al. (NA3 Collaboration), Z. Phys. C 11, 195 (1981).

13. S. Falciano et al. (NA10 Collaboration), Z. Phys. C 31, 513 (1986); M. Guanziroli et al. (NA10 Collaboration), Z. Phys. C 37, 545 (1988).

14. J.S. Conway et al. (E615 Collaboration), Phys. Rev. D 39, 92 (1989).

15. A. Brandenburg, O. Nachtmann, and E. Mirkes, Z. Phys. C 60, 697 (1993).

16. D. Boer, A. Brandenburg, O. Nachtmann, and A. Utermann, Eur. Phys. J. C40, 55 (2005).

17. O. Nachtmann and A. Reiter, Z. Phys. C 24, 283 (1984); G.W. Botz, P. Haberl, and O. Nachtmann, Z. Phys. C 67, 143 (1995).

18. D. Boer, Phys. Rev. D 60, 014012 (1999).

19. D. Boer and P.J. Mulders, Phys. Rev. D 57, 5780 (1998).

20. B. Pasquini and P. Schweitzer, Phys. Rev. D 90, 014050 (2014).

21. C.P. Chang and H.N. Li, Phys. Lett. B 726, 262 (2013).

22. L.Y. Zhu et al. (FNAL E866/NuSea Collaboration), Phys. Rev. Lett. 99, 082301 (2007) and Phys. Rev. Lett. 102, 182001 (2009).

23. E. Anassontzis et al. (E537 Collaboration), Phys. Rev. D 38, 1377 (1988). 